\title{
Improving the Robustness of Vector Fitting to Outliers in the Data
}

\author{
D. Deschrijver, L. Knockaert, T. Dhaene \\ Dept. of Information Technology (INTEC) \\ Ghent University - IBBT, Sint Pietersnieuwstraat 41, 9000 Ghent, Belgium \\ \{dirk.deschrijver, luc.knockaert, tom.dhaene\}@intec.ugent. be
}

\begin{abstract}
This letter introduces a new algorithm for broadband macromodeling of passive electronic components from frequency response data. It modifies the Vector Fitting algorithm in such a way that the $L_{1}$ norm of the approximation error is minimized, rather than the $L_{2}$ norm. It is shown that this approach is more robust towards outliers in the data.
\end{abstract}

\section{Introduction}

Robust broadband macromodeling techniques are of crucial importance for efficient time domain and frequency domain simulation of high-speed interconnect structures. The standard Vector Fitting (VF) algorithm starts from an S-parameter frequency response, sampled at a discrete set of frequencies. It computes a macromodel by minimizing a weighted iterative cost function in the $L_{2}$ sense [1]. In real-life situations, the quality of the $L_{2}$ fitting model may be degraded due to outliers in the data. An outlier is a value in the data that deviates strongly from the other values, and is usually caused by measurement or instrumentation errors. This paper proposes a modified Vector Fitting algorithm that minimizes the $L_{1}$ norm of the complex fitting error instead [2]. Numerical results illustrate that the new approach is more robust with respect to outliers [3].

\section{Vector Fitting Technique}

\subsection{Modeling Algorithm}

Given a discrete set of S-parameter data samples $\left\{s_{k}=j \omega_{k}, H\left(s_{k}\right)\right\}_{k=0}^{K}$, a rational macromodel with numerator $N^{t}(s)$ and denominator $D^{t}(s)$ can be computed 
iteratively $(t=1, \ldots, T)$ by successively solving least squares problems $[1,4]$

$$
\arg \min J=\sum_{k=0}^{K}\left|w\left(s_{k}\right)\right|^{2}\left|\sigma^{t}\left(s_{k}\right) H\left(s_{k}\right)-(\sigma H)^{t}\left(s_{k}\right)\right|^{2}
$$

As in [5], both $(\sigma H)^{t}(s)$ and $\sigma^{t}(s)$ belong to a linear span of $P$ rational basis functions $\Psi\left(s, a_{p}^{t-1}\right)=\left(s-a_{p}^{t-1}\right)^{-1}$ that are based on the previously identified poles $a_{p}^{t-1}$. Note that the initial poles $a_{p}^{0}$ are chosen by a heuristical scheme [1]

$$
\begin{gathered}
(\sigma H)^{t}(s)=\frac{N^{t}(s)}{D^{t-1}(s)}=c_{0}^{t}+\sum_{p=1}^{P} c_{p}^{t} \Psi\left(s, a_{p}^{t-1}\right) \\
\sigma^{t}(s)=\frac{D^{t}(s)}{D^{t-1}(s)}=1+\sum_{p=1}^{P} \tilde{c}_{p}^{t} \Psi\left(s, a_{p}^{t-1}\right)
\end{gathered}
$$

In this letter, it is shown that the $L_{1}$ norm can be minimized by selecting the user-defined frequency-dependent weighting factor $w(s)$ in (1) as

$$
w(s)=\frac{\sqrt{\left|H(s)-H^{t-1}(s)\right|}}{H(s)-H^{t-1}(s)}
$$

where $H^{t-1}(s)=N^{t-1} / D^{t-1}$ denotes the model response at iteration step $t-1$.

\subsection{Proof Outline}

First, define the auxiliary function $f(s)$ as

$$
f(s)=\frac{\left|D^{t}(s) H(s)-N^{t}(s)\right|^{2}}{\left|D^{t-1}(s) H(s)-N^{t-1}(s)\right|^{2}}
$$

Applying the weighting factor $w(s)$ in (4) to the cost function $J$ in (1) yields

$$
\arg \min J=\sum_{k=0}^{K}\left|H\left(s_{k}\right)-H^{t-1}\left(s_{k}\right)\right| f\left(s_{k}\right)
$$

Hence, upon convergence of the iterative scheme $\left(D^{t-1} \rightarrow D^{t}\right.$ and $\left.N^{t-1} \rightarrow N^{t}\right)$, it follows that $f(s) \rightarrow 1$. Therefore, it is clear that cost function $J$ in (6) effectively minimizes the $L_{1}$ norm of the complex fitting error $\left\|H(s)-H^{t-1}(s)\right\|_{1}$.

\section{Example : Quarter Wavelength Filter}

The reflection coefficient $S_{11}$ of a 2-port microwave quarter wavelength filter is computed over the frequency range $[1 \mathrm{GHz}-12 \mathrm{GHz}]$. Suppose that, due to 
inaccuracies in the data acquisition, the S-parameter response contains 6 outlying data samples which are marked by black arrows. All data samples are modelled by a rational 28-pole strictly proper transfer function using the proposed fitting methodology ( $L_{1}$ norm) and the standard Vector Fitting algorithm ( $L_{2}$ norm).

The resulting macromodels are shown as a solid red curve in Figs. 1 and 2, and the data samples are marked as blue dots. It is clear from Fig. 1 that the $L_{1}$ norm approximation yields an overall accurate result, and is not much affected by the presence of the outliers. On the other hand, the outliers lead to a strong degradation of the model quality for the $L_{2}$ norm approximation, as shown in Fig. 2. This observation is confirmed by Fig. 3 where the maximum absolute fitting error of both fitting models is shown as a function of the frequency.

\section{Conclusion}

An iterative algorithm is proposed for $L_{1}$ norm identification of broadband macromodels from S-parameter data. It is shown that the method is more robust when the frequency response contains outliers. The effectiveness of the approach is illustrated by applying it to a quarter wavelength filter.

\section{Acknowledgment}

This work was supported by the Research Foundation Flanders (FWO-V).

\section{References}

[1] B. Gustavsen, A. Semlyen, Rational Approximation of Frequency Domain Responses by Vector Fitting, IEEE Trans. on Power Delivery, vol. 14, no. 1, pp. 1052-1061, 1999.

[2] C. Burrus, J. Barreto, I. Selesnick, Iterative Reweighted Least-Squares Design of FIR Filters, IEEE Trans. on Signal Processing. vol. 42, no. 11, pp. 2926-2936, 1994.

[3] A. Kijko, Seismologic Outliers: $L_{1}$ or Adaptive $L_{p}$ Norm Application, Bulletin of the Seismologic Society of America, vol. 84, no. 2, pp. 473-477, 1994.

[4] D. Deschrijver, Gustavsen, T. Dhaene, Advancements in Iterative Methods for Rational Approximation in the Frequency Domain, IEEE Trans. on Power Delivery, vol. 22, no. 3, pp. 1633-1642, 2007. 
[5] D. Deschrijver, B. Haegeman, T. Dhaene, Orthonormal Vector Fitting : a Robust Macromodeling Tool for Rational Approximation of Frequency Domain Responses, IEEE Trans. on Advanced Packaging, vol. 33, no. 2, pp. 216-225, 2007.

Fig 1 : Magnitude of $S_{11}$ : model $L_{1}$ (red) and data (blue)

Fig 2 : Magnitude of $S_{11}$ : model $L_{2}$ (red) and data (blue)

Fig 3 : Absolute fitting error of model $L_{1}$ (green) and model $L_{2}$ (blue) 

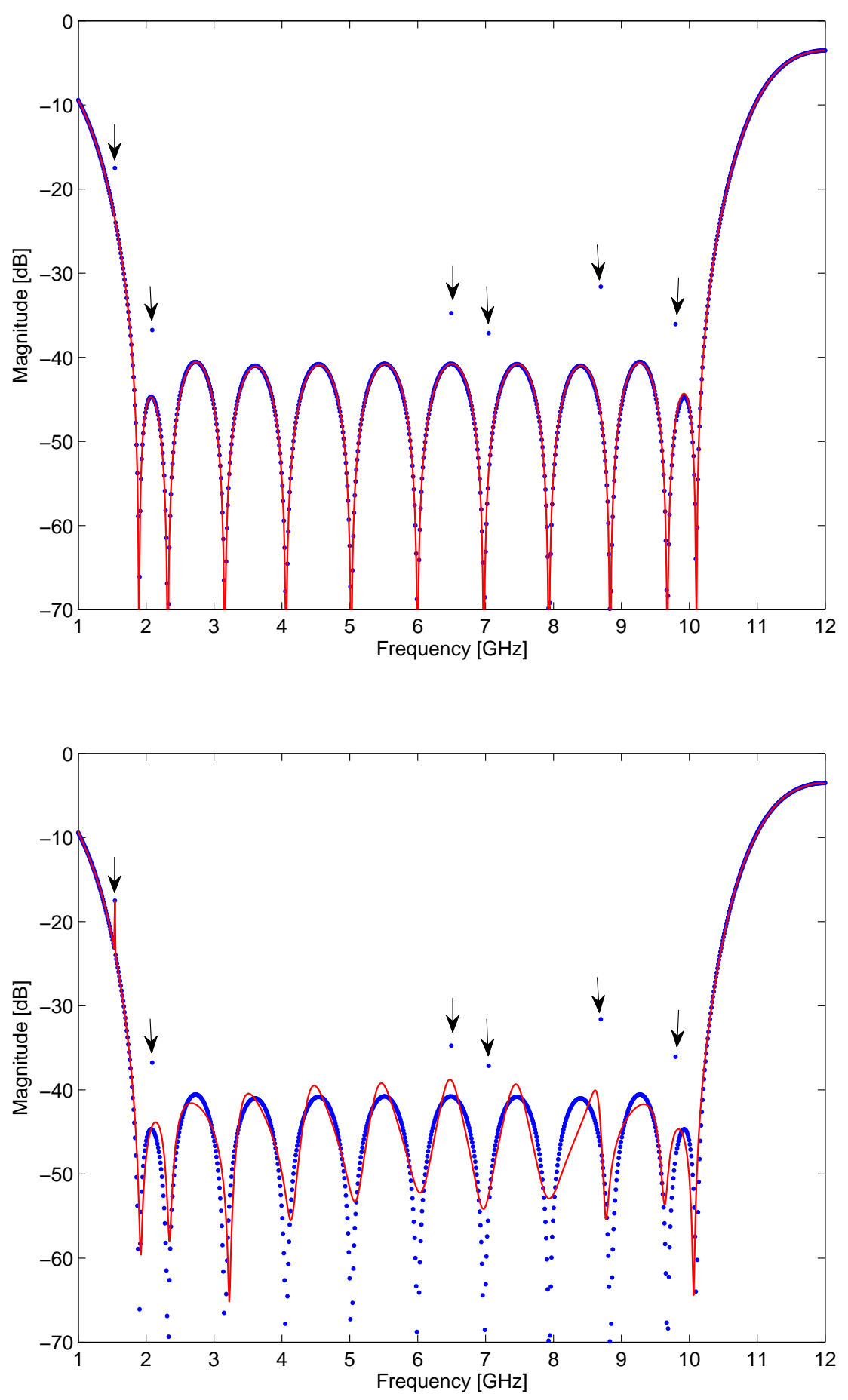


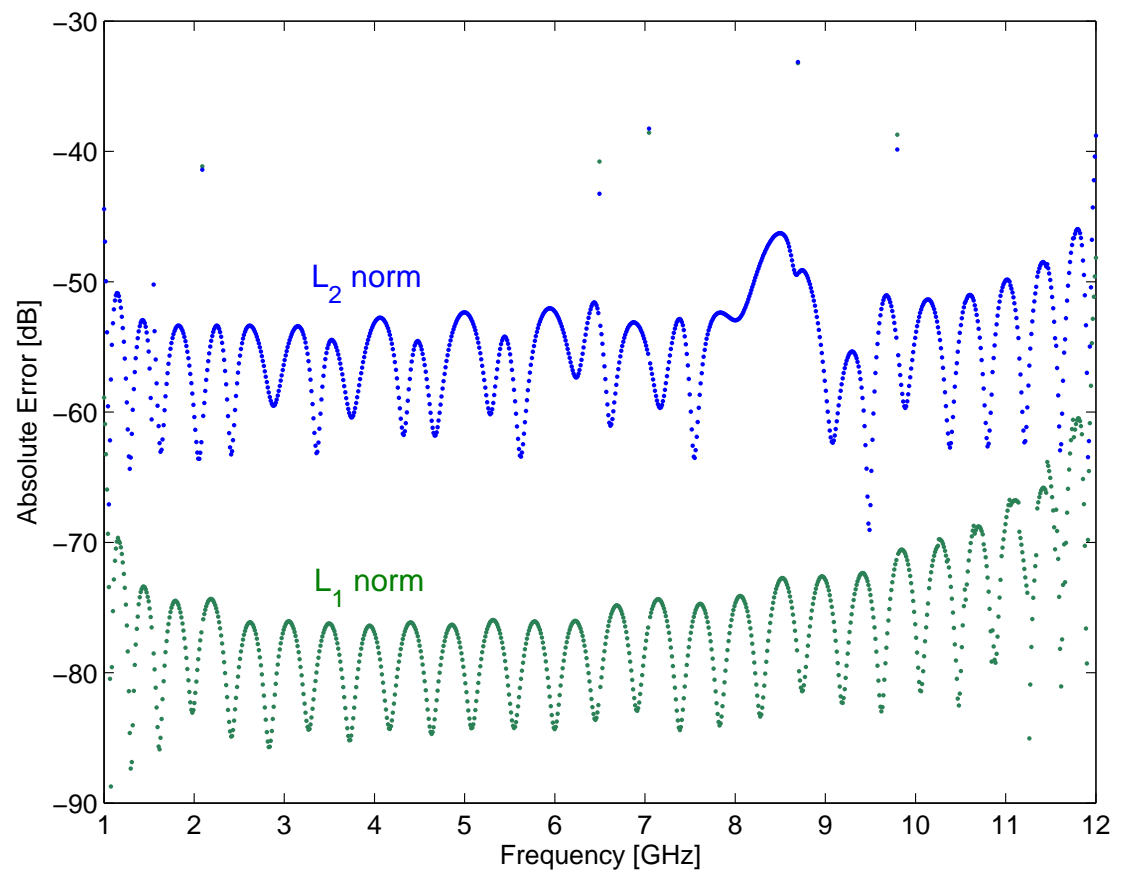

